\title{
Differential proteomic analysis of tibial subchondral bone from male and female guinea pigs with spontaneous osteoarthritis
}

\author{
YING WANG $^{1}$, CHENGAI WU $^{1}$, JIANFENG TAO ${ }^{1}$, DANHUI ZHAO $^{2}$, XU JIANG ${ }^{3}$ and WEI TIAN ${ }^{4}$ \\ ${ }^{1}$ Department of Molecular Orthopedics and ${ }^{2}$ Animal Laboratory, Beijing Research Institute of Traumatology and \\ Orthopedics; Departments of ${ }^{3}$ Orthopedics and ${ }^{4}$ Spine Surgery, Beijing Jishuitan Hospital, Beijing 100035, P.R. China
}

Received September 8, 2020; Accepted March 17, 2021

DOI: 10.3892/etm.2021.10065

\begin{abstract}
A proteomic study on the tibial subchondral bone in guinea pigs with spontaneous osteoarthritis was performed to investigate the molecular alterations that occur in early osteoarthritis. A total of 132 healthy Hartley guinea pigs (aged 1 month; 66 female and 66 male) were randomly divided into 11 groups of six. Changes in articular cartilage and tibial subchondral bone were assessed using macroscopic examinations and micro-computed tomography. iTRAQ-integrated liquid chromatography-tandem mass spectrometry was used to identify differentially altered proteins in the tibial subchondral bone between 1- and 3-month-old guinea pigs, which were then validated using western blotting. A gradual progression of cartilage degeneration was observed in the knee joints of the subject animals from 5-11 months. With aging, the tibial subchondral trabecular bone acquired more plate-like and less anisotropic properties, with increased bone mineral density, bone volume, trabecular thickness and numbers. The proteomic study identified 138 and 113 proteins significantly differentially expressed between 3- and 1-month old guinea pigs in both the male and female animals, respectively. Western blotting confirmed the increased expression of osteoblast-associated protein S100 calcium-binding protein A8 (S100A8) and the deregulated expression of osteoclast-associated proteins coronin 1A (CORO1A) and T-cell immune regulator 1 (TCIRG1) in the 3-month old guinea pigs in comparison to the 1-month old guinea pigs. Spontaneous cartilage degeneration
\end{abstract}

Correspondence to: Professor Chengai Wu, Department of Molecular Orthopedics, Beijing Research Institute of Traumatology and Orthopedics, 31 Xinjiekou East Street, Xicheng, Beijing 100035, P.R. China

E-mail: chengai_Wu@163.com

Abbreviations: BMD, bone mineral density; DA, degree of anisotropy; LC-MS/MS, liquid chromatography tandem-mass spectrometry; OA, osteoarthritis; SMI, structure model index; $\mathrm{Tb}$. Pf, trabecular bone pattern factor; Tb. Sp, trabecular separation; VOI, volume of interest

Key words: osteoarthritis, Hartley guinea pig, subchondral bone, proteomics in the knee joints of male Hartley guinea pigs tended to be more serious compared with the females during the development of osteoarthritis. Together, the results suggest that osteoblast-associated protein S100A8 and osteoclast-associated proteins CORO1A and TCIRG1 are potentially key regulators of early osteoarthritic development in tibial subchondral bone.

\section{Introduction}

Osteoarthritis (OA) is a chronic and progressive disorder of the joints, characterized by a gradual loss of articular cartilage and remodeling of the underlying bone. OA of the knee, the most common type of $\mathrm{OA}$, is one of the fastest growing causes of disability, and affected $>250$ million individuals worldwide (1). Despite its high prevalence, there is a lack of effective pharmacological interventions for treatment or management of OA at present and the most common treatment strategies are pain relief or total knee replacement (2). Thus far, pharmaceutical treatment strategies for OA have relied on the use of analgesics and non-steroidal anti-inflammatory drugs (3). Whilst research into disease-modifying drugs for OA that may delay disease progression by targeting various signaling pathways involved in the pathogenesis of OA is currently underway $(2,4)$, the development of tissue-specific disease-modifying treatments for knee OA has been plagued with difficulties (5), primarily due to a lack of clear understanding of the molecular mechanisms underlying the initiation and development of this disease.

Knee OA is a multifactorial joint disease that involves various anatomical structures of the joint, including the articular cartilage, subchondral bone, synovial membrane, ligaments and menisci (6,7). A key factor that may contribute to OA onset and development is the biomechanical characteristics of the subchondral bone. Indeed, changes in the architecture and composition of the subchondral bone have been frequently recognized in animal OA models and patients with OA. These changes include abnormal bone turnover, sclerosis of the subchondral plate, osteophyte formation, thinning of the trabecular bone, subchondral cysts and bone marrow lesions $(8,9)$. These microstructural changes are highly associated with subchondral bone stiffening and degeneration of the overlying cartilage (10). Amelioration of the abnormalities in subchondral bone may not only alleviate joint pain but may also delay cartilage degeneration (11), making 
subchondral bone-targeted treatment a promising approach for the treatment of OA. However, a comprehensive investigation into the potential role of subchondral bone changes in OA and the related underlying molecular mechanisms is still required.

Due to the difficulties in monitoring the initiation and progression of OA in human patients, animal models with naturally occurring articular disorders similar to human $\mathrm{OA}$ are instead widely used to investigate the pathogenesis of this disease (12). The Hartley strain of guinea pigs can spontaneously develop an OA-related phenotype of the knee joint. In Hartley guinea pigs, the articular cartilage exhibits age-dependent degenerative changes, for example, a gradual decrease in the number of chondrocytes and amount of proteoglycan that closely resembles what is observed in human OA. In addition, Hartley guinea pigs share certain OA risk factors with humans, such as age, sex and body weight $(13,14)$. All of these advantages make Hartley guinea pigs a suitable animal model for studying the molecular mechanisms underlying OA initiation and progression. Previous studies have focused on altered gene-expression patterns in osteoarthritic articular cartilage, whereas research concerning the association of subchondral bone with OA remains limited $(15,16)$. Thus, determining the molecular mechanisms underlying abnormal alterations in the subchondral bone in a Hartley guinea pig model of knee OA may not only increase knowledge of the pathogenesis of OA, but also highlight potential therapeutic targets for novel OA disease-modifying drugs.

Liquid chromatography tandem-mass spectrometry (LC-MS/MS) combined with iTRAQ technology is widely used to identify new biomarkers and pathways involved in several diseases. However, to the best of our knowledge, there are no studies examining the molecular changes in subchondral bone in OA using large-scale proteomic analysis. To address this gap in the literature, the objective of the present study was to identify potentially novel molecular biomarkers associated with the early pathological process of OA using the established Hartley guinea pig model of spontaneous knee OA. Differentially expressed proteins were analyzed and three candidates in subchondral bone tissues derived from male and female Hartley guinea pigs in the very early phase of OA were identified by performing iTRAQ-based LC-MS/MS. The proteomic characterization of osteoarthritic subchondral bone allowed for a deeper understanding of this disease and may facilitate future development of nonsurgical treatments for OA.

\section{Materials and methods}

Animal handling and gross examination. Male $(\mathrm{n}=66)$ and female ( $n=66$ ) Hartley guinea pigs (age, 1 month old; mean weight, $328 \mathrm{~g}$ for female animals, $374 \mathrm{~g}$ for male animals) were obtained from Beijing Vital River Laboratory Animal Technology Co., Ltd.. Animals were randomly assigned to groups of six and housed together in plastic cages, measuring $90 \times 60 \times 15 \mathrm{~cm}$ with a stainless-steel grid lid, and maintained at a temperature of $25^{\circ} \mathrm{C}$, a relative humidity of $55 \pm 10 \%$ and a $12 \mathrm{~h}$ light/dark cycle. The animals were provided with ad libitum access to water and standard rodent chow. All animal experiments were approved by the Beijing Jishuitan Hospital Animal Care and Use Committee and were performed in accordance with the institutional guidelines for care and use of animals.
The general design of the study is shown in Fig. 1A. Animals were randomly selected for sacrifice at 1-month intervals for 11 months, 6 animals at a time. The body weights of all animals were recorded before sacrifice. Articular cartilage was collected immediately after guinea pigs were sacrificed by cervical dislocation under anesthesia using $3 \%$ sodium pentobarbital (30 mg/kg body weight) intraperitoneally. After sacrifice the knee-joints were disarticulated, the femurs and tibias were separated, the articular cartilage of the femur and the tibia were cleaned with $0.9 \%$ normal saline and the gross visual appearance of the articular cartilage was recorded using a Canon EOS 40D digital camera with a $50-\mathrm{mm}$ lens (Canon Inc.).

Microstructural analysis of subchondral bone. The microarchitecture of whole knee-joint specimens was analyzed using a SkyScan 1172 micro-computed tomography (CT) system (SkyScan; Bruker Corporation). The microfocus X-ray source was set at $86 \mathrm{kV}$ and $116 \mu \mathrm{A}$, with a pixel size of $12 \mu \mathrm{m}$. After scanning, 3D reconstructions with a voxel size of $12 \mu \mathrm{m}$ were acquired using NRecon version 1.6.6.15 (SkyScan; Bruker Corporation). For analysis of the subchondral trabecular bone, a cuboid of trabecular bone measuring $2.05 \times 2.05 \times 0.6 \mathrm{~mm}^{3}$ was selected as the volume of interest (VOI). After VOI selection, the images were binarized using the following gray-level thresholds in Hounsfield units: 105-252 for ages 1-2 months; 120-252 for ages 3-6 months; 125-252 for age 7 months; and 130-252 for ages 8-11 months. The binarized images were used to calculate morphological parameters using CTAn version 1.14.4 (SkyScan; Bruker Corporation). The bone volume fraction (BV/TV as a percentage), trabecular thickness (Tb. Th, mm), trabecular number (Tb. N, 1/mm), structure model index (SMI), trabecular bone pattern factor (Tb. Pf, $1 / \mathrm{mm}$ ), degree of anisotropy (DA) and trabecular separation (Tb. Sp, mm) were calculated. Additionally, the bone mineral density (BMD, $\mathrm{g} / \mathrm{cm}^{3}$ ) was calibrated using the attenuation coefficients of two hydroxyapatite phantom materials with known mineral densities of 0.25 and $0.75 \mathrm{~g} / \mathrm{cm}^{3}$.

Protein digestion and iTRAQ labeling. Protein digestion was performed using the filter-aided sample-preparation procedure described by Wisniewski et al (17). The mixture of peptides generated was labeled with 4-plex iTRAQ reagents, according to the manufacturer's protocol (Applied Biosystems; Thermo Fisher Scientific, Inc.). Briefly, $200 \mu \mathrm{g}$ total protein for each tibial subchondral bone sample was incorporated into a $30 \mu 1$ solution containing $4 \%$ sodium dodecyl sulfate, $100 \mathrm{mM}$ dithiothreitol, and $100 \mathrm{mM}$ Tris- $\mathrm{HCl}$ ( $\mathrm{pH}$ 8.0). After heating at $95^{\circ} \mathrm{C}$ for $5 \mathrm{~min}$ and then cooling to room temperature, each sample was filtered using an ultrafiltration filter (pore size, $10 \mathrm{kDa}$; Sartorius AG) and treated with $200 \mu \mathrm{l}$ UT buffer (8 $\mathrm{M}$ urea and $150 \mathrm{mM}$ Tris- $\mathrm{HCl}$ at $\mathrm{pH} 8.0$ ). Subsequently, $100 \mu 150 \mathrm{mM}$ iodoacetamide in UT buffer was added to block reduced cysteines, and the samples were incubated at room temperature for $20 \mathrm{~min}$ in the dark. The filters were washed three times with $100 \mu \mathrm{l}$ UT buffer and then twice with $100 \mu \mathrm{l}$ dissolution buffer $(50 \mathrm{mM}$ triethylammonium bicarbonate at $\mathrm{pH}$ 8.5). Finally, the protein suspensions were digested overnight with $40 \mu \mathrm{l}$ trypsin (Promega Corporation) buffer $(2 \mu \mathrm{g}$ trypsin in $40 \mu \mathrm{l}$ dissolution buffer) at $37^{\circ} \mathrm{C}$. The generated 


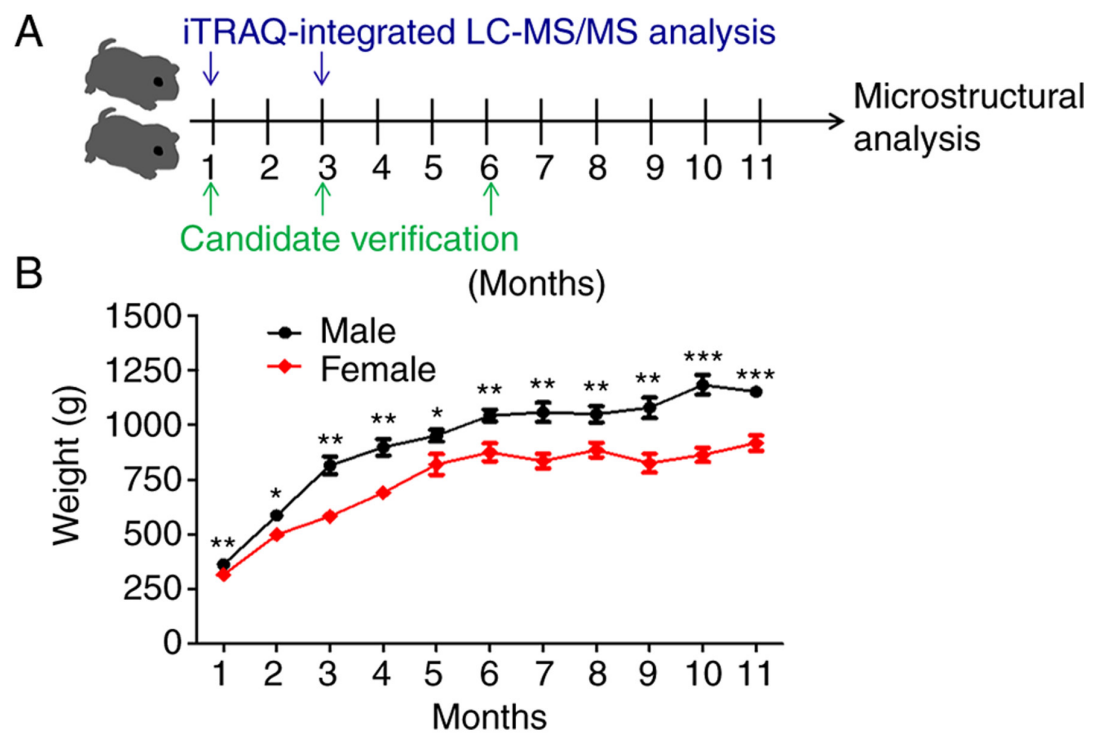

Figure 1. Flow chart of the study design. (A) A flow chart of the design of the present study. (B) Comparison of body weights between male and female Hartley guinea pigs for each time point. Male animals showed significantly faster growth rates compared with the female guinea pigs. LC-MS/MS, liquid chromatography tandem mass spectrometry. ${ }^{*} \mathrm{P}<0.05,{ }^{* *} \mathrm{P}<0.01,{ }^{* * *} \mathrm{P}<0.001$ vs. female.

peptide concentrations were estimated using UV light spectral density at $280 \mathrm{~nm}$. For labeling, each iTRAQ reagent was dissolved in $70 \mu \mathrm{l}$ ethanol and added to the respective mixture of peptides. The peptides obtained from the tibial subchondral bone samples from the guinea pigs were named as follows: 1-month-old male guinea pigs (1MM)-1, -2 and -3; 1-month-old female guinea pigs (1MF)-1, -2 and -3 ; 3-month-old male guinea pigs (3MM)-1, -2 and -3 ; and 3 -month-old female guinea pigs (3MF)-1, -2 and -3 . Tibial subchondral bone samples from the $1 \mathrm{MM}, 1 \mathrm{MF}, 3 \mathrm{MM}$ and $3 \mathrm{MF}$ groups were labeled with isobaric iTRAQ tags of masses 114, 115, 116 and $117 \mathrm{Da}$, respectively, and were multiplexed and vacuum dried.

Peptide fractionation using strong cationic-exchange chromatography. The iTRAQ-labeled peptides were fractionated using strong cationic-exchange (SCX) chromatography performed on the AKTA Purifier system (GE Healthcare). The dried peptide mixture was reconstituted and acidified with $2 \mathrm{ml}$ buffer A consisting of $10 \mathrm{mM} \mathrm{KH}_{2} \mathrm{PO}_{4}$ in $25 \%(\mathrm{v} / \mathrm{v})$ of acetonitrile ( $\mathrm{pH} 2.7$ ), and then subjected to SCX fractionation on a PolySULFOETHYL column $(4.6 \times 100 \mathrm{~mm}, 5 \mu \mathrm{m}$, $200 \AA$ A; PolyLC Inc.). The peptides were eluted at a flow rate of $1 \mathrm{ml} / \mathrm{min}$ with a gradient of $0-10 \%$ buffer B (buffer A with $500 \mathrm{mM} \mathrm{KCl}$ added) for $2 \mathrm{~min}, 10-20 \%$ buffer B for $25 \mathrm{~min}, 20-45 \%$ buffer B for $5 \mathrm{~min}$ and $50-100 \%$ buffer B for $5 \mathrm{~min}$. The elution was monitored using the absorbance at $214 \mathrm{~nm}$ and fractions were collected every min. Finally, $\sim 30$ collected fractions were combined into 10 pools and desalted on $\mathrm{C} 18$ Cartridges [Empore ${ }^{\mathrm{TM}}$ SPE Cartridges C18 (standard density), bed inner diameter $7 \mathrm{~mm}$, volume $3 \mathrm{ml}$; Sigma-Aldrich; Merck KGaA]. Each SCX salt step fraction was concentrated by vacuum centrifugation and then reconstituted in $40 \mu 10.1 \%(\mathrm{v} / \mathrm{v})$ acetic acid. All samples were stored at $-80^{\circ} \mathrm{C}$ until required for LC-MS/MS analysis.

LC-MS/MS analysis. Experiments were performed on a $\mathrm{Q}$ Exactive mass spectrometer coupled with an EasynLC(Proxeon
Biosystems; Thermo Fisher Scientific, Inc.). For each fraction, $5 \mu \mathrm{g}$ peptide mixture was subjected to nano-LC-MS/MS analysis. The peptide mixture was loaded onto a reverse phase trap column (Thermo Fisher Scientific, Inc.; Acclaim PepMap100, $100 \mu \mathrm{m} \times 2 \mathrm{~cm}$, nanoViper C18) connected to a C18-reversed phase analytical column (Thermo Fisher Scientific, Inc.; Easy Column; $10-\mathrm{cm}$ long, $75-\mu \mathrm{m}$ inner diameter, $3-\mu \mathrm{m}$ resin) in buffer $\mathrm{C}(0.1 \%$ formic acid $)$ and separated with a linear gradient of buffer D (buffer $\mathrm{C}$ with $84 \%$ acetonitrile added) at a flow rate of $300 \mathrm{nl} / \mathrm{min}$ controlled using IntelliFlow technology over $120 \mathrm{~min}$. MS data were acquired using a data-dependent top 10 method that dynamically selected the most abundant precursor ions from the survey scan (300-1,800 m/z) for higher energy collisional dissociation (HCD) fragmentation. The target value was determined based on predictive automatic gain control. The dynamic exclusion duration was $40 \mathrm{sec}$. Survey scans were acquired at a resolution of 70,000 at $\mathrm{m} / \mathrm{z} 200$, and the resolution for $\mathrm{HCD}$ spectra was set to 17,500 at $\mathrm{m} / \mathrm{z} 200$; the isolation width was $2 \mathrm{~m} / \mathrm{z}$. The normalized collision energy was $30 \mathrm{eV}$, and the underfill ratio was defined as $0.1 \%$ on $\mathrm{Q}$ Exactive.

Sequence database examination and data analysis. MS/MS spectra were searched using the MASCOT engine (Matrix Science; version 2.2) embedded into Proteome Discoverer version 1.4 (Thermo Fisher Scientific, Inc.) against the Uniprot Cavia porcellus database (20,415 sequences, downloaded on October 10th, 2015) and a decoy database. For protein identification, the following parameters were used: Peptide mass tolerance, $20 \mathrm{ppm}$; fragment mass tolerance, 0.1 Da; enzyme, trypsin; maximum missed cleavages, 2; fixed modifications, carbamidomethyl (C), iTRAQ4plex (K), and iTRAQ4plex (N-term); variable modification, oxidation (M); peptide false discovery rate $(\mathrm{FDR}) \leq 0.01$.

Gene ontology (GO) annotation and Kyoto encyclopedia of genes and genomes (KEGG) pathway analysis. GO analysis 
1-month 2-months 3-months 4-months 5-months 6-months 7-months 8 -months 9-months 10-months 11-months

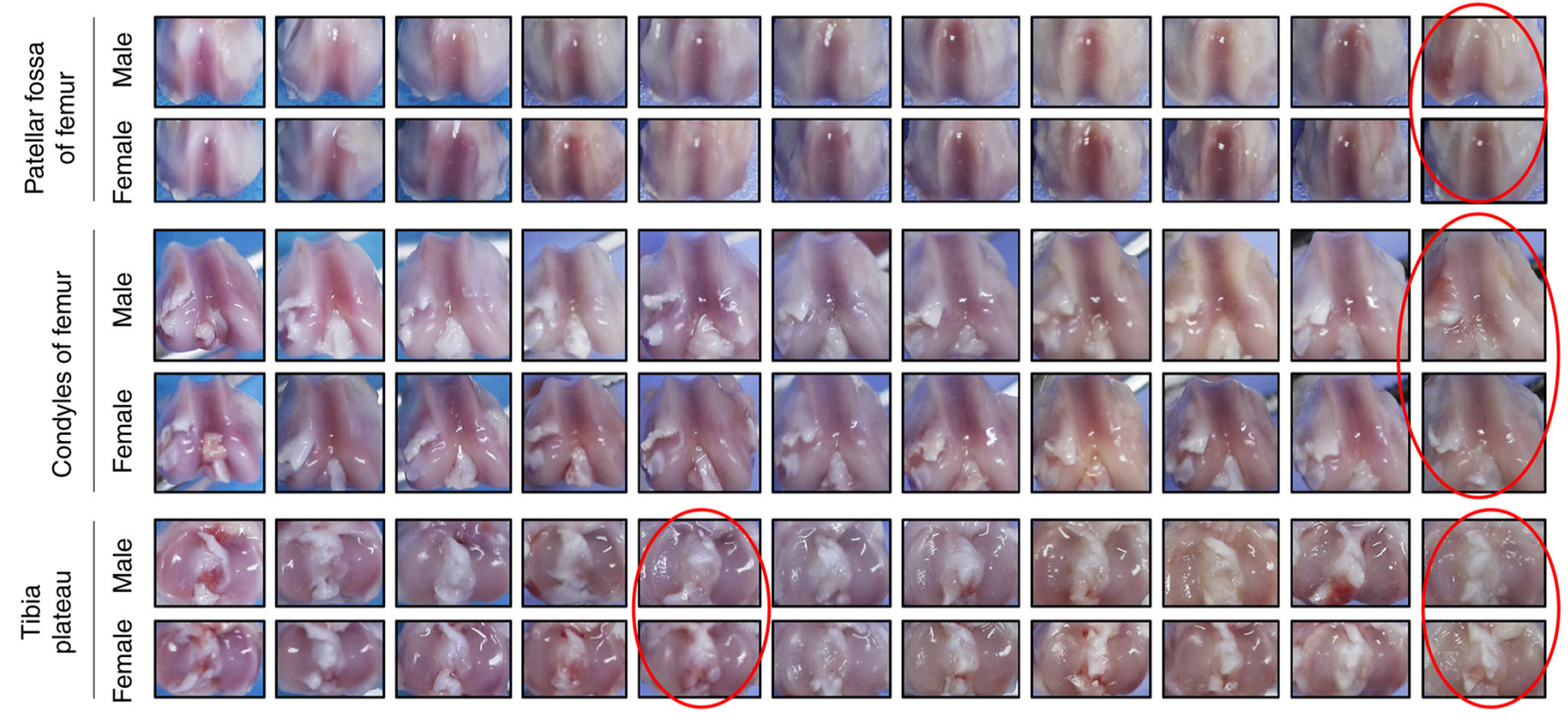

Figure 2. Macroscopic progression of cartilage degeneration in the knee joints of male and female Hartley guinea pigs aged 1-11 months. Articular cartilage damage initially (at 5 months of age) appeared in the area of the medial tibial plateau that was not covered by the meniscus in both male and female Hartley guinea pigs. Compared with females, the cartilage on the medial tibial plateau (the part not covered by the medial meniscus) of male guinea pigs underwent significant degeneration, which manifested as a rough cartilage surface, cartilage softening and erosion (red circle). The degree of articular cartilage degeneration increased with age, starting from 5 months in both male and female animals. At 11 months of age, cartilage degeneration was serious and had invaded the medial and lateral areas of the tibial plateau, femoral condyle and patellar fossa. The degree of cartilage degeneration of male guinea pigs was more severe than that of females (red circle). Representative images of femoral condyles, patellar fossas and tibial plateaus are shown.

was performed to annotate the identified differentially expressed proteins. Firstly, the protein sequences of the differentially expressed proteins identified were retrieved in batches from the UniProtKB database (https://www.uniprot.org/) in the FASTA format. Then, the functional GO annotations and classifications of all the differentially expressed proteins were applied using the Blast2GO program. Biological pathway enrichment analysis was performed based on KEGG pathway analysis. The FASTA sequences of all the differentially expressed proteins were compared against the online KEGG database (http://www.genome.ad.jp/kegg/) and mapped to the pathways included in KEGG. The corresponding KEGG pathways were subsequently extracted.

Western blot analysis. At sacrifice, samples of the right tibial subchondral bone were collected for western blot analysis. For coronin 1A (CORO1A), S100 calcium-binding protein A8 (S100A8) and TCIRG1 protein expression studies, $100 \mu \mathrm{g}$ total protein from tibial subchondral bone was resolved using a 4-12\% SDS-PAGE gel. After transfer to PVDF membranes, the membranes were blocked for $1 \mathrm{~h}$ at room temperature in $1 \mathrm{X}$ PBS containing $0.15 \%$ Tween-20 (1X PBST) and 5\% non-fat dry milk, and subsequently immunoblotted with the indicated primary antibodies in 1X PBST with $5 \%$ non-fat dry milk at $4{ }^{\circ} \mathrm{C}$ overnight. The primary antibodies included anti-CORO1A (Aviva Systems Biology, Corp; cat. no. ARP38899_T100; 1:400), anti-S100A8 (Abcam; cat. no. ab92331; 1:1,000) and anti-TCIRG1 (Santa Cruz Biotechnology, Inc.; cat. no. sc-162300; 1:500). Signals were visualized using enhanced chemiluminescence reagent (Thermo Fisher Scientific, Inc.) and horseradish peroxidase
HRP-conjugated goat anti-rabbit IgG (Abcam; cat. no. ab97051; 1:5,000), HRP-conjugated rabbit anti-mouse IgG (Abcam; cat. no. ab6728; 1:5,000) and HRP-conjugated rabbit anti-goat IgG (Abcam; cat. no. ab6741; 1:5,000) secondary antibodies for $2 \mathrm{~h}$ at room temperature. $\beta$-actin (Abcam; cat. no. ab6276; $1: 1,000)$ was used as the internal control.

Statistical analysis. SAS version 9.2 (SAS Institute, Inc.) was used for statistical analyses for between-group differences. GraphPad Prism version 8.0 (GraphPad Software, Inc.) was used for statistical analyses of differences between sexes. Differences between ages in BMD, BV/TV, Tb. Th, Tb. N, SMI, Tb. Pf, DA and Tb. Sp values of the subchondral trabecular bone were analyzed using a non-parametric Kruskal-Wallis test, and a pair-wise comparison among 11 ages was performed for each parameter based upon Dunn's post hoc test. Sex differences in BMD, BV/TV, Tb. Th, Tb. N, SMI, Tb. Pf, DA and $\mathrm{Tb}$. Sp values were analyzed using a Student's t-test or non-parametric Mann Whitney U test. $\mathrm{P}<0.05$ was considered to indicate a statistically significant difference.

\section{Results}

Gross morphological observation of spontaneous degeneration of articular cartilage. After an initial sharp increase from 1 to 5 months, the weight of all guinea pigs remained relatively stable over time. Compared with the female guinea pigs, male animals showed a significantly faster growth rate until the age of 11 months (Figs. 1B and S1). Macroscopic articular cartilage lesions initially appeared in the region of the medial tibial plateau not covered by the meniscus in both male and female 
A
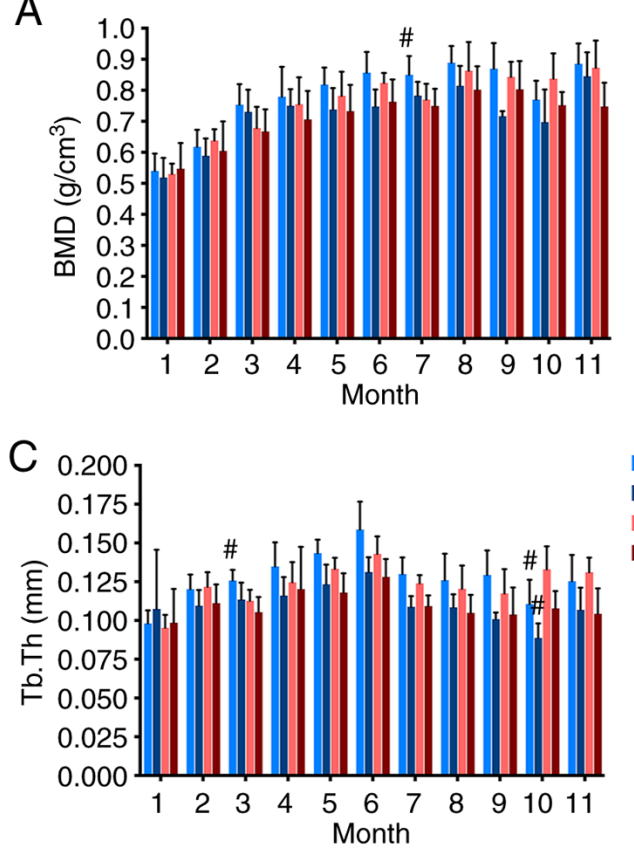

E

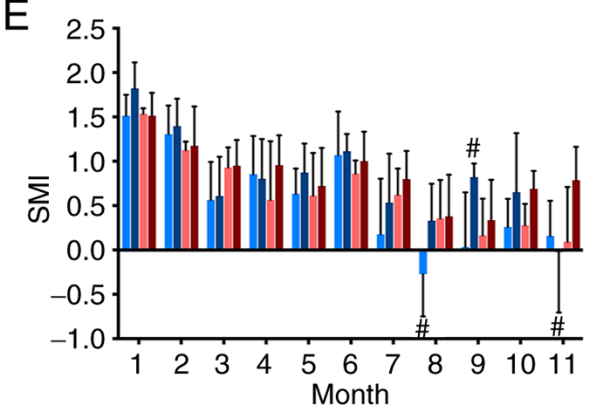

G

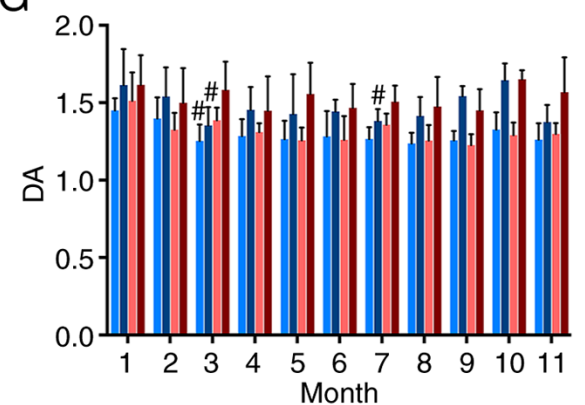

- Female lateral ${ }^{*}$



Male medial ${ }^{2}$

Male lateral ${ }^{*}$
Female medial ${ }^{*}$

- Female lateral
- Male medial ${ }^{\star}$

- Male lateral*

- Female medial $^{\star}$

B


$\mathrm{F}$

- Male medial*

- Male lateral ${ }^{*}$

- Female medial ${ }^{*}$

- Female lateral ${ }^{\star}$

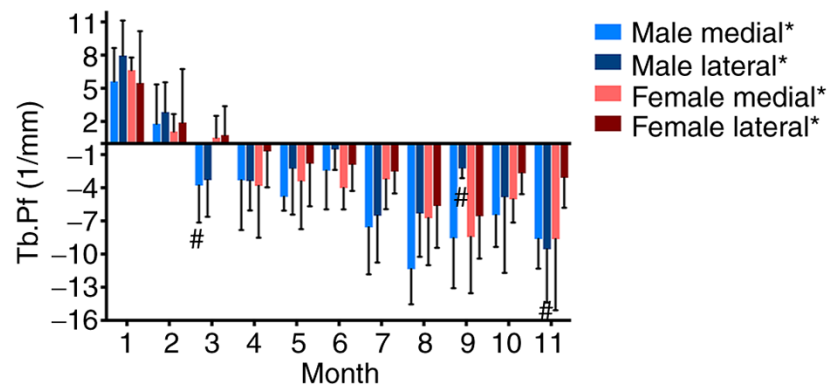

$\mathrm{H}$

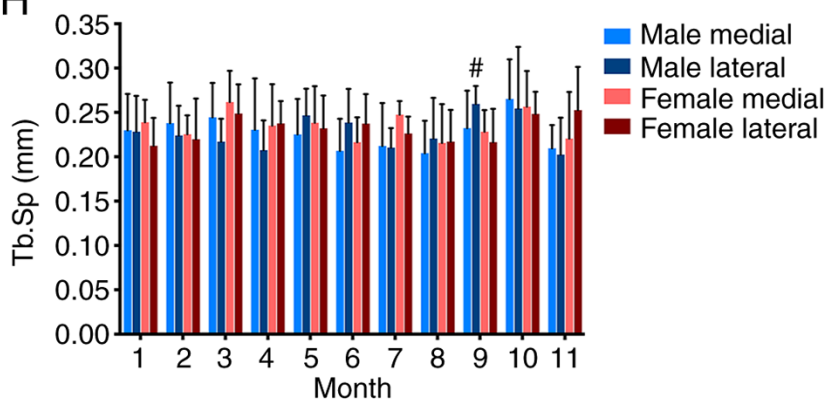

Figure 3. Age and sex related micro-architectural alterations in the tibial subchondral trabecular bone in Hartley guinea pigs. Detailed microstructural changes in both the medial and lateral sides of subchondral trabecular bone in male and female guinea pigs are shown. (A) BMD. (B) BV/TV. (C) Tb. Th. (D) Tb. N. (E) SMI. (F) Tb. Pf. (G) DA. (H) Tb. Sp. Data are presented as the mean \pm standard deviation. BV/TV, bone volume fraction; Tb. Th, trabecular thickness; Tb. $\mathrm{N}$, trabecular number; SMI, structure model index; Tb. Pf, trabecular bone pattern factor; DA, degree of anisotropy; Tb. Sp, trabecular separation; BMD, the bone mineral density. ${ }^{~} \mathrm{P}<0.05$ vs. female. "Significant differences were observed between ages in the same sex and detailed comparison results are available in Table SI-II.

Hartley guinea pigs at the age of 5 and 11 months (red circle; Fig. 2). The degeneration of the articular cartilage worsened with age, starting at 5 months. By the age of 11 months, the degenerative cartilage lesions were noticeable and had invaded all aspects of the medial and lateral compartments of the tibial plateau in addition to the condyles and patellar fossa of the femur. Moreover, spontaneous cartilage degeneration in the knee joints tended to be more serious in male Hartley guinea pigs compared with female Hartley guinea pigs.
Micro-CT analysis of subchondral trabecular bone. To further characterize the microarchitecture of the subchondral trabecular bone in the guinea pigs, Micro-CT analysis for both sexes was performed (Fig. 3). The BMD of the medial tibial trabecular bone showed an age-related increase in both male and female guinea pigs from 1 to 6 months of age (Fig. 3A). After 6 months, BMD values exhibited slight fluctuations; however, the differences between ages were not significant (Fig. 3A). A similar tendency was observed 
A



B

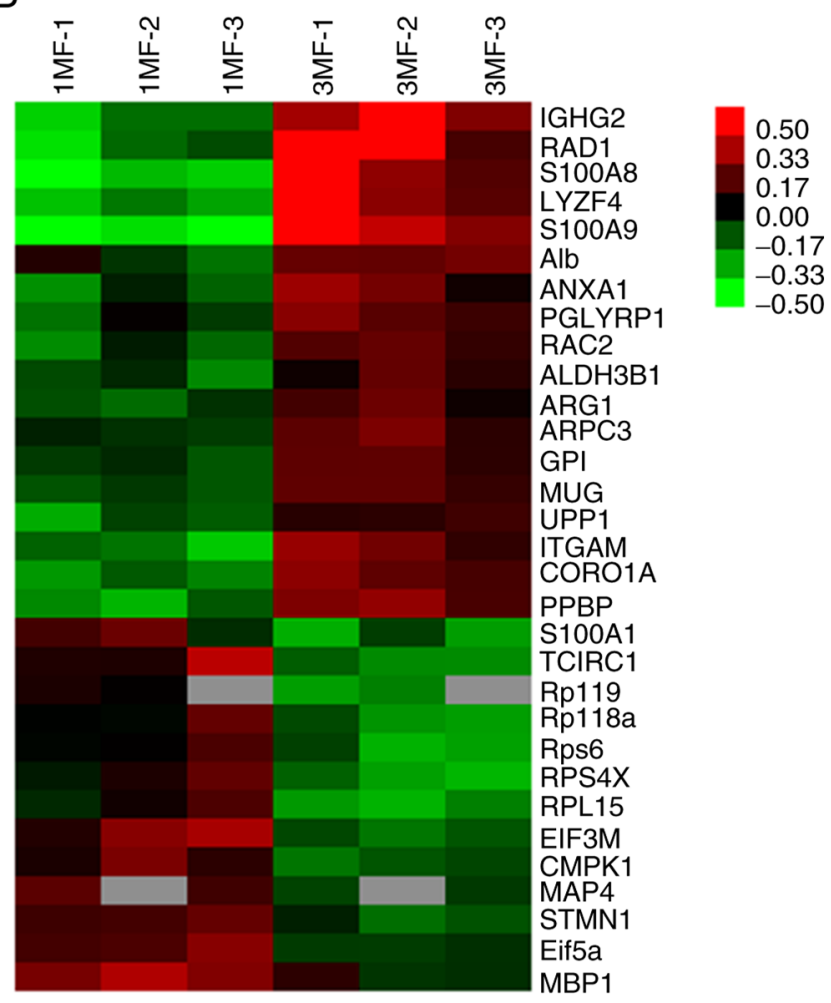

Figure 4. Heat maps of significantly altered proteins with definite identities detected in the subchondral bone of (A) male and (B) female Hartley guinea pigs. A total of 24 upregulated and 16 downregulated proteins were identified in male guinea pigs, and 18 upregulated and 13 downregulated proteins were identified in female guinea pigs. Upregulated and downregulated proteins are shown as red and green spots, respectively. Grey regions indicate that data is not available. 1MM, 1-month-old male guinea pig; 1MF, 1-month-old female guinea pig-3MM, 3-month-old male guinea pig; 3MF, 3-month-old female guinea pig.

for the lateral tibial trabecular bone in female guinea pigs. However, the BMD of the lateral tibial trabecular bone in male animals showed a gradual upward trend from ages 1 to 4 months, followed by temporary fluctuations. The $\mathrm{BV} / \mathrm{TV}$ increased over time on both the medial and lateral sides in the female animals, peaking at 6 months (Fig. 3B). The pattern of BV/TV variation in the medial trabecular bone of male animals was consistent with that in the female animals. In both male and female guinea pigs, the thickness of the trabecular bone gradually increased from 1 to 6 months, and then declined. The trabecular number in the subchondral trabecular bone showed a slight increasing trend (Fig. 3C and D). Trabecular SMI and Tb. Pf exhibited a general decreasing trend with a slight increase during the middle stage of growth (Fig. 3E and F). With age, the subchondral trabecular bone became less anisotropic. No significant changes were observed for trabecular separation as the animals aged (Fig. 3G and $\mathrm{H}$ ). These results showed that spatial and temporal ultrastructural changes occur in the subchondral trabecular bone of both male and female guinea pigs during aging (detailed comparison results are shown in Tables SI and II).
Identification of differentially expressed proteins in tibial subchondral bone. To identify proteomic differences in the tibial articular subchondral bone of both male and female guinea pigs during the early growth stages, high-accuracy LC-MS/MS integrated with iTRAQ analysis was used to determine which proteins were differentially expressed between 1 and 3 months of age. A total of 12,313 unique peptides matching 2,316 non-redundant proteins were identified in the tibial articular subchondral bone of guinea pigs with high confidence ( $\geq 1$ unique peptide with an FDR $\leq 1 \%$ ). The list of candidates was narrowed down to 138 proteins in male guinea pigs and 113 proteins in female guinea pigs using a threshold of $>1.2$-fold change and $\mathrm{P}<0.05$. After characterizing the expression patterns of these differentially expressed proteins, the proteins were further grouped into two clusters: Upregulated and downregulated expression. Amongst these, half of them were upregulated (69/138) and the other half (69/138) downregulated in the male animals, whereas 60 out of 113 proteins were upregulated and 53 proteins (53/113) were downregulated in the female animals (Fig. S2). Two independent heat maps were produced to show the accumulation patterns of these differentially modulated identity-determined proteins found 
A

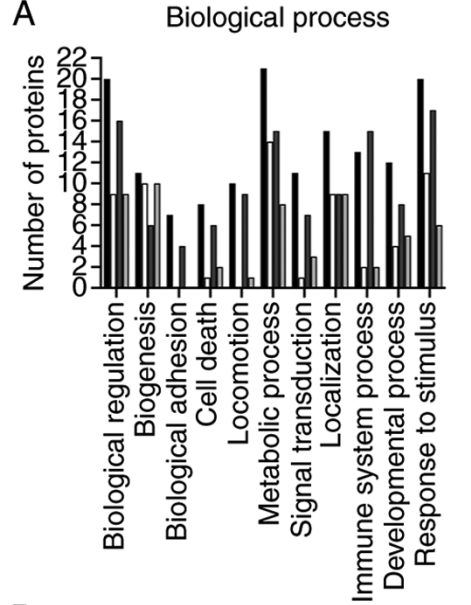

B



C



Figure 5. Differentially expressed proteins with definite identities were grouped according to GO category. Numbers of differentially expressed proteins associated with GO terms in the GO categories (A) biological process, (B) molecular function and (C) cellular component GO. All data are presented on the basis of GO second-level terms. The numbers of upregulated and downregulated proteins for each category are shown. GO, Gene Ontology.

in the tibial articular subchondral bone tissues from both male and female guinea pigs between different age groups (Fig. 4).

Functional classification and Kyoto encyclopedia of genes and genomes $(K E G G)$ analysis of differentially regulated proteins. To further understand these differentially expressed proteins identified by the iTRAQ analysis, the proteomic results were analyzed using Gene Ontology (GO), a universally recognized and standardized classification system for gene function. However, since only a small percentage of the guinea pig proteome has been characterized in the Uniprot database, this classification places a particular emphasis on proteins with definite identities. With this caveat, 40 proteins (24 upregulated and 16 downregulated) were identified in male guinea pigs and 31 proteins (18 upregulated and 13 downregulated) were identified in female animals. These proteins were subsequently annotated as three key categories; namely, biological processes, molecular function and cellular components based on GO enrichment analysis. These proteins were mapped onto 11 biological functional categories and annotated with 10 molecular functional terms (Fig. 5A and B). These differentially expressed proteins were also mapped onto 7 cellular components annotations: 'Macromolecular complex' (male, 10, 25.0\%; female, 16, 51.6\%), 'membrane-enclosed lumen' (male, 3, 7.5\%; female, 5, 16.1\%), 'organelle' (male, 15, 37.5\%; female, 25, 80.6\%), 'cell junction' (male, 4, 10.0\%; female, 6, 19.4\%), 'cell' (male, 16, 40.0\%; female, 25, 80.6\%), 'membrane' (male, 10, 25.0\%; female, 16, 51.6\%), and 'extracellular region' (male, 13, 32.5\%; female, 22, 71.0\%) (Fig. 5C). The numbers of upregulated and downregulated proteins for each category are shown in Fig. 5.

To further investigate the functions and signaling pathways in which these identified proteins were involved in, KEGG-based pathway analysis was performed. Several important biological pathways that may be potentially affected by the differentially expressed proteins with definite identities are summarized in Table I. These results reveal that protein signature can be alerted in tibial subchondral bone.

Verification of differentially expressed proteins using western blotting. To validate the key proteomic changes identified by the untargeted iTRAQ-based proteomic analysis, western blotting was used to assess the expression of selected candidates. Specific attention was given to those proteins involved in the processes of bone formation and resorption, as these may serve a major role in OA bone remodeling (18). Expression of S100A8, CORO1A and TCIRG1, possibly affect the process of bone remodeling (19-21) (Fig. 6A). As shown in Fig. 6B, the western blotting analysis results precisely mirrored the proteomic findings. During OA progression, there was a continued increase in the expression of CORO1A and S100A8 in the subchondral bone in both male and female guinea pigs from 1 to 6 months. Following an initial decrease, the abundance of TCIRG1 increased from months 3 to 6 in female guinea pigs, whereas its expression levels remained relatively stable in male guinea pigs. Together the data suggest that these proteins may serve a role in subchondral bone homeostasis during spontaneous OA.

\section{Discussion}

Involvement of the subchondral bone in the pathogenesis of OA has been increasingly recognized by recent advances in research. Alterations in the metabolic properties of the subchondral bone during OA progression are more poignant than secondary manifestations and may serve as the potential cause of the disease $(18,22-25)$. In the present study, it was 
Table I. Kyoto Encyclopedia of Genes and Genomes analysis of the differentially regulated proteins with known identities in the tibial articular subchondral bone of guinea pigs.

\section{Pathway term}

'Ribosome'

'Phagosome'

'Carbon metabolism'

'Rap1 signaling pathway'

'Natural killer cell mediated cytotoxicity'

'Glycolysis / Gluconeogenesis'

'Ras signaling pathway'

'Chemokine signaling pathway'

'PI3K-Akt signaling pathway'

'Lysosome'

'Regulation of autophagy'
Associated proteins
H0W636, H0VST8, H0W953, H0V1L6, H0VUY3, H0UY44

H0V1Y3, H0VVX0, P11578, H0UW57

H0VLR7, H0UXA8, H0VCM3, H0VZ24

H0VVP9, H0VVX0, P11578

H0VVP9, H0VDD2, H0VVX0

H0VCM3, H0VKB0

A9LS48, H0VVP9

A9LS48, H0WD04

A9LS48, H0V1L6

H0VDY4, H0UW57

H0V7Z3

A
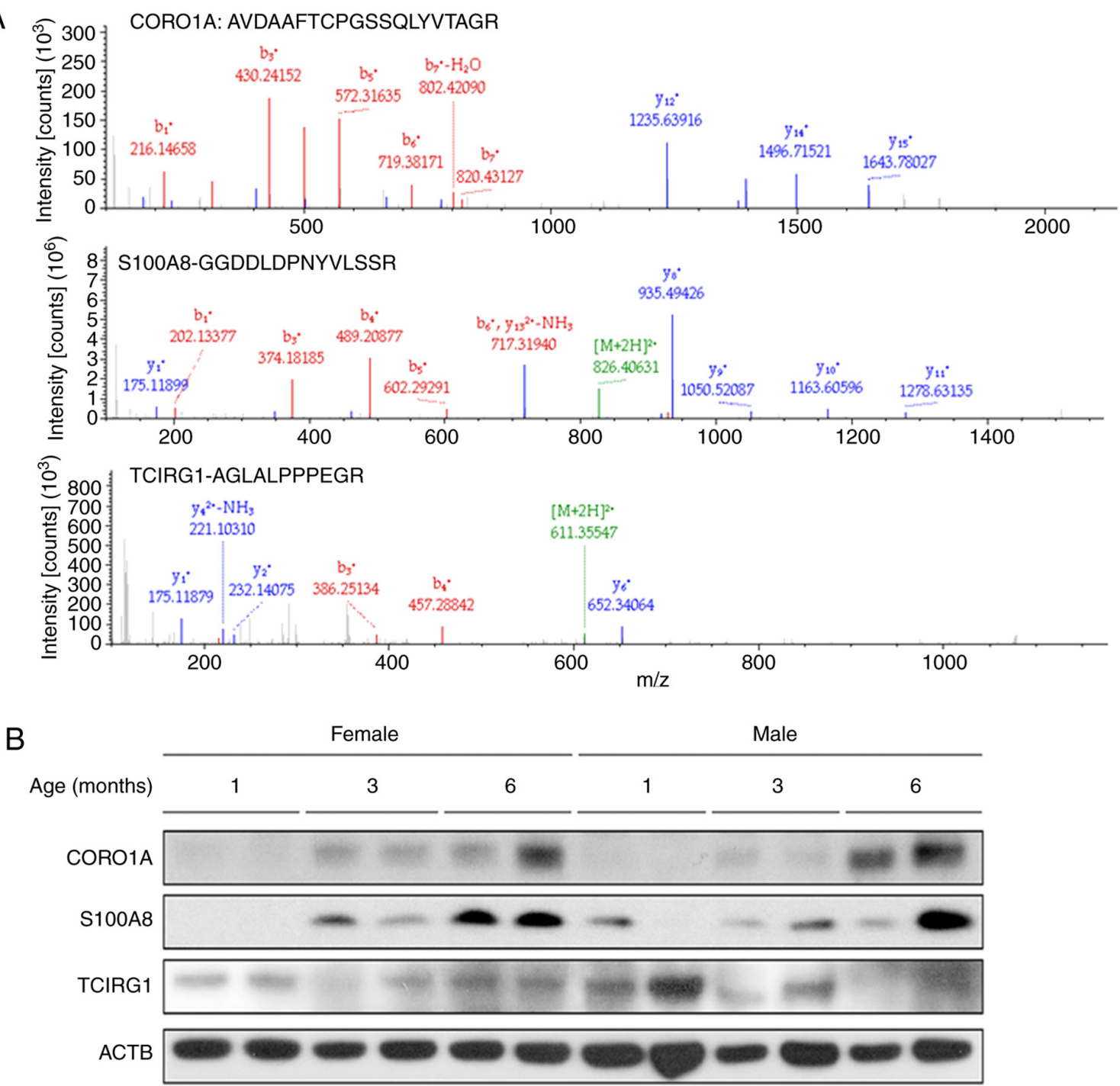

Figure 6. Validation of the three candidate differentially expressed proteins, CORO1A, S100A8 and TCIRG1, by western blotting. (A) Representative mass spectra of CORO1A, S100A8 and TCIRG1-derived peptides (AVDAAFTCPGSSQLYVTAGR, GGDDLDPNYVLSSR and AGLALPPPEGR, respectively) isolated from subchondral bone tissues from 3-month old guinea pigs. (B) Protein samples extracted from the subchondral bone tissues of the indicated age groups were subjected to western blotting. There was a continuous increase in the expression of CORO1A and S100A8 in the subchondral bone in both male and female guinea pigs from 1 to 6 months. Following an initial decrease, the abundance of TCIRG1 increased from month 3 to 6 in the female guinea pigs, whereas its expression levels remained relatively stable in the male guinea pigs. ACTB was used as the loading control. ACTB, $\beta$-actin; S100A8, S100 calcium-binding protein $\mathrm{A} 8$; CORO1A, coronin 1A; TCIRG1, T-cell immune regulator 1. 
confirmed that both male and female Hartley guinea pigs could be used as a suitable model to investigate the etiology and pathogenesis of OA, consistent with previous studies (26-28). More importantly, to the best of our knowledge, the present study was the first to document a differentially modulated proteome in the tibial articular subchondral bone in male and female guinea pigs using an unbiased proteomic approach followed by western blotting for confirmation. The identification of critical proteins using mass spectrometry-based strategies will serve a key role in elucidating the underlying mechanism of OA, which will not only facilitate further investigation of the pathological process of OA development, but also help develop novel nonsurgical treatment strategies for management of this disease.

In patients with OA, the degree of subchondral trabecular remodeling is correlated with body weight (13). A similar positive correlation between body weight and BMD of subchondral trabecular bone was observed in our 11-month longitudinal study of cartilage degeneration and bone remodeling in male and female guinea pigs. Additionally in the present study, whilst cartilage degeneration occurred in both male and female guinea pigs, male animals tended to exhibit more severe pathological alterations, which may be attributable to their faster growth rate, resulting in their reaching greater body weights earlier than the female counterparts (26).

Research findings from both clinical and animal studies indicate that the pathological remodeling of the subchondral bone occurs in the early stage of OA progression, prior to cartilage degeneration (13,29-32). Consistent with these findings, it was shown that elevated bone turnover occurred as early as 2 months of age, prior to cartilage degeneration in both male and female Hartley guinea pigs. In the early growth stage, it is normally expected that the tibial subchondral trabecular bone shows an age-related increase in BMD, bone volume, trabecular thickness and trabecular number, indicating continual bone remodeling. However, the subchondral trabecular bone in the present study was shown to be more plate-like, as indicated by the continuous decrease in SMI and Tb. Pf., unlike what is observed during the normal aging process (33). Changes from rod-like to plate-like morphology may increase the stiffness of the subchondral trabecular bone (34), which is detrimental to the overlying articular cartilage. Additionally, the subchondral trabecular bone was found to be inferior in its ability to transfer load from cartilage, based on the evidence of lower DA. Moreover, the medial side of the subchondral trabecular bone had a lower SMI and Tb. Pf compared with the lateral sides in both male and female guinea pigs. Such differences between the medial and lateral compartments of the tibial plateau indicate increased mechanical strength on the medial side, which may explain why cartilage degeneration initially occurred in the medial tibial plateau, particularly in the region not covered by the meniscus, as observed in both our current and previous study (26).

A decrease in $\mathrm{Tb}$. $\mathrm{N}$ and an increase in $\mathrm{Tb}$. Sp in subchondral trabeculae have been reported in OA (35). However, an increasing trend in $\mathrm{Tb}$. $\mathrm{N}$ and no significant difference in Tb. Sp was observed based on the micro-CT analysis in the present study. This discrepancy could be due to factors such as individual variances between animals, or differences in diet and housing conditions (36). Such variations can interfere with disease development and lead to inconsistencies between OA animal experiments (36). In the present study, this issue was circumvented by employing a non-targeted proteomic approach using iTRAQ-integrated LC-MS/MS technology. Instead of investigating only one or a few specific proteins at a time, it was possible to simultaneously identify multiple proteins with this unbiased approach. Non-targeted methods not only directly determine the expression levels of proteins but also provide insights into the functional properties of a larger number of potential candidates under both physiological and pathological conditions. Whilst previous studies $(15,16,37)$ have revealed a large number of cytokines and proteases in the articular cartilage, synovial fluid and serum in patients with OA and in animal models, limited information is currently available regarding the protein profile in the osteoarthritic subchondral bone (38). Microarray technology has revealed the gene-expression profile of the subchondral bone obtained from the knee joint of an experimental rat OA model (39), demonstrating the importance and necessity of research on subchondral bone using high-throughput protein analysis. In the present study, iTRAQ-based LC-MS/MS technology was used to identify proteins involved in the development of $\mathrm{OA}$ in the subchondral bone using a spontaneous guinea pig OA model.

The present proteomic analysis showed that 6.6 and $8 \%$ of all proteins were significantly altered in the tibial subchondral bone of female and male guinea pigs, respectively, suggesting that further analysis of these differentially expressed proteins may increase our understanding of the role these subchondral bone microstructural changes may serve in OA. Owing to the lack of information on the proteome of guinea pigs, it was only possible to identify 40 proteins (24 upregulated and 16 downregulated) and 31 proteins (18 upregulated and 13 downregulated) with characterized identity, amongst these significantly altered proteins in male and female guinea pigs, respectively.

Bone remodeling is a physiological process governed by an equilibrium between bone formation through osteoblast activity and bone resorption through osteoclast activity (40). Disruption of this balance, which may be caused by chronic inflammation (41), may lead to abnormal changes in bone architecture and quality. OA subchondral bone impairment is characterized by abnormal bone remodeling and altered osteoblast-osteoclast coupling. Based on the present and previous studies $(13,29-31)$, during the pathogenesis of OA, abnormal remodeling of the subchondral bone occurs in the early stage prior to cartilage degeneration, suggesting a vital role of subchondral bone remodeling in OA onset and development. As a result, proteins that are associated with either bone formation or bone resorption have been the subjects of extensive research. From the analysis of differentially expressed proteins identified in the proteomic profiling in the present study, it was decided to focus on three particular proteins, S100A8, CORO1A and TCIRG1, which exhibited a high association with biological processes GO terms, including 'bone formation' and 'bone resorption'. The expression of these proteins was first verified using western blotting. S100A8, which is associated with osteoblast differentiation (19) and osteoarthritic osteophyte formation (42), was upregulated in the osteoarthritic subchondral bone of both male and female 
guinea pigs. CORO1A, a negative regulator of bone resorption activity through inhibition of the release of cathepsin $\mathrm{K}$, is an important factor in bone resorption by degrading bone matrix proteins (20). The abundance of CORO1A was shown to continuously increase with age in the present study. TCIRG1, a component of the osteoclast vacuolar proton pump, was downregulated at 3 months of age in both male and female guinea pigs, followed by a distinct expression pattern at 6 months of age in guinea pigs of both sexes. Increasing evidence has shown that mutational inactivation of TCIRG1 causes autosomal recessive osteopetrosis, a disorder of bone metabolism caused by subnormal osteoclast function (43-45). In addition, osteoclast function can be restored via lentiviral vector-mediated overexpression of TCIRG1 (21). These results further add to the increasing amount of evidence that these proteins may mediate the abnormal formation of subchondral bone in the early growth stage. In future studies, it will be both interesting and important to elucidate their specific functions in initiation and progression of OA.

In conclusion, the results of the present study provide novel insights into the potential mechanisms underlying the malformation of subchondral bone during OA development and progression. Future studies using various cell lines, animal models and human osteoarthritic specimens are required to further elucidate the roles of these differentially expressed proteins in the osteoarthritic subchondral bone identified in the present study, as they may provide a mechanistic basis for development of novel OA treatments.

\section{Acknowledgements}

Not applicable.

\section{Funding}

This study was supported by grants from the National Natural Science Foundation of China (grant nos. 81330043 and 81071499).

\section{Availability of data and materials}

The datasets generated and/or analyzed during the current study are not publicly available due to compliance with the Inner Confidentiality Policy of Scientific Data by Beijing Research Institute of Traumatology and Orthopedics but are available from the corresponding author on reasonable request.

\section{Authors' contributions}

CW, XJ and WT designed the experiments. YW performed the animal experiments. YW, XJ and CW analyzed the data. DZ facilitated animal breeding, acquired images and interpreted the data. JT performed image analysis. YW and CW wrote the manuscript. CW, YW and XJ confirm the authenticity of all the raw data. All authors read and approved the final manuscript.

\section{Ethics approval and consent to participate}

All animal experiments were approved by the Beijing Jishuitan Hospital Animal Care and Use Committee, Beijing, China.

\section{Patient consent for publication}

Not applicable.

\section{Competing interests}

The authors declare that they have no competing interests.

\section{References}

1. Hunter DJ and Bierma-Zeinstra S: Osteoarthritis. Lancet 393: 1745-1759, 2019.

2. Mobasheri A: The future of osteoarthritis therapeutics: Targeted pharmacological therapy. Curr Rheumatol Rep 15: 364, 2013.

3. Roman-Blas JA, Bizzi E, Largo R, Migliore A and Herrero-Beaumont G: An update on the up and coming therapies to treat osteoarthritis, a multifaceted disease. Expert Opin Pharmacother 17: 1745-1756, 2016.

4. Parrish WR, Byers BA, Su D, Geesin J, Herzberg U, Wadsworth S, Bendele A and Story B: Intra-articular therapy with recombinant human GDF5 arrests disease progression and stimulates cartilage repair in the rat medial meniscus transection (MMT) model of osteoarthritis. Osteoarthritis Cartilage 25: 554-560, 2017.

5. Wei Y and Bai L: Recent advances in the understanding of molecular mechanisms of cartilage degeneration, synovitis and subchondral bone changes in osteoarthritis. Connect Tissue Res 57: 245-261, 2016.

6. Neogi T: Clinical significance of bone changes in osteoarthritis. Ther Adv Musculoskelet Dis 4: 259-267, 2012.

7. Martel-Pelletier J and Pelletier JP: Is osteoarthritis a disease involving only cartilage or other articular tissues? Eklem Hastalik Cerrahisi 21: 2-14, 2010.

8. Muratovic D, Cicuttini F, Wluka A, Findlay D, Wang Y, Otto S, Taylor D, Humphries J, Lee Y, Labrinidis A, et al: Bone marrow lesions detected by specific combination of MRI sequences are associated with severity of osteochondral degeneration. Arthritis Res Ther 18: 54, 2016.

9. Audrey HX, Abd Razak HR and Andrew TH: The truth behind subchondral cysts in osteoarthritis of the knee. Open Orthop J 8: 7-10, 2014.

10. Day JS, Ding M, van der Linden JC, Hvid I, Sumner DR and Weinans H: A decreased subchondral trabecular bone tissue elastic modulus is associated with pre-arthritic cartilage damage. J Orthop Res 19: 914-918, 2001.

11. Yu D, Xu J, Liu F, Wang X, Mao Y and Zhu Z: Subchondral bone changes and the impacts on joint pain and articular cartilage degeneration in osteoarthritis. Clin Exp Rheumatol 34: 929-934, 2016.

12. Hunter DJ and Little CB: The great debate: Should osteoarthritis research focus on 'mice' or 'men'? Osteoarthritis Cartilage 24: 4-8, 2016.

13. Reina N, Cavaignac E, Pailhé R, Pailliser A, Bonnevialle N, Swider P and Laffosse JM: BMI-related microstructural changes in the tibial subchondral trabecular bone of patients with knee osteoarthritis. J Orthop Res 35: 1653-1660, 2017.

14. van der Kraan P, Matta C and Mobasheri A: Age-related alterations in signaling pathways in articular chondrocytes: Implications for the pathogenesis and progression of osteoarthritis - a mini-review. Gerontology 63: 29-35, 2017.

15. Lourido L, Calamia V, Mateos J, Fernández-Puente P, Fernández-Tajes J, Blanco FJ and Ruiz-Romero C: Quantitative proteomic profiling of human articular cartilage degradation in osteoarthritis. J Proteome Res 13: 6096-6106, 2014.

16. Tsolis KC, Bei ES, Papathanasiou I, Kostopoulou F, Gkretsi V, Kalantzaki K, Malizos K, Zervakis M, Tsezou A and Economou A: Comparative proteomic analysis of hypertrophic chondrocytes in osteoarthritis. Clin Proteomics 12: 12, 2015.

17. Wisniewski JR, Zougman A, Nagaraj N and Mann M: Universal sample preparation method for proteome analysis. Nat Methods 6: 359-362, 2009.

18. Zhu X, Chan YT, Yung PSH, Tuan RS and Jiang Y: Subchondral bone remodeling: A therapeutic target for osteoarthritis. Front Cell Dev Biol 8: 607764, 2021.

19. Zreiqat H, Howlett CR, Gronthos S, Hume D and Geczy CL: S100A8/S100A9 and their association with cartilage and bone. J Mol Histol 38: 381-391, 2007. 
20. Ohmae S, Noma N, Toyomoto M, Shinohara M, Takeiri M, Fuji H, Takemoto K, Iwaisako K, Fujita T, Takeda N, et al: Actin-binding protein coronin 1A controls osteoclastic bone resorption by regulating lysosomal secretion of cathepsin K. Sci Rep 7: 41710, 2017.

21. Moscatelli I, Thudium CS, Flores C, Schulz A, Askmyr M, Gudmann NS, Andersen NM, Porras O, Karsdal MA Villa A, et al: Lentiviral gene transfer of TCIRG1 into peripheral blood CD34(+) cells restores osteoclast function in infantile malignant osteopetrosis. Bone 57: 1-9, 2013.

22. Chen Y, Hu Y, Yu YE, Zhang X, Watts T, Zhou B, Wang J, Wang T, Zhao W, Chiu KY, et al: Subchondral trabecular rod loss and plate thickening in the development of osteoarthritis. J Bone Miner Res 33: 316-327, 2018.

23. Ma L, Zhao X, Liu Y, Wu J, Yang X and Jin Q: Dihydroartemisinin attenuates osteoarthritis by inhibiting abnormal bone remodeling and angiogenesis in subchondral bone. Int J Mol Med 47: 1, 2021

24. Cui Z, Crane J, Xie H, Jin X, Zhen G, Li C, Xie L, Wang L, Bian Q, Qiu T, et al: Halofuginone attenuates osteoarthritis by inhibition of TGF- $\beta$ activity and H-type vessel formation in subchondral bone. Ann Rheum Dis 75: 1714-1721, 2016.

25. Finnilä MAJ, Thevenot J, Aho OM, Tiitu V, Rautiainen J, Kauppinen S, Nieminen MT, Pritzker K, Valkealahti M, Lehenkari P and Saarakkala S: Association between subchondral bone structure and osteoarthritis histopathological grade. J Orthop Res 35: 785-792, 2017.

26. Bendele AM: Animal models of osteoarthritis. J Musculoskelet Neuronal Interact 1: 363-376, 2001.

27. Lampropoulou-Adamidou K, Lelovas P, Karadimas EV, Liakou C, Triantafillopoulos IK, Dontas I and Papaioannou NA: Useful animal models for the research of osteoarthritis. Eur J Orthop Surg Traumatol 24: 263-271, 2014.

28. Kuyinu EL, Narayanan G, Nair LS and Laurencin CT: Animal models of osteoarthritis: Classification, update, and measurement of outcomes. J Orthop Surg Res 11: 19, 2016

29. Wang T, Wen CY, Yan CH, Lu WW and Chiu KY: Spatial and temporal changes of subchondral bone proceed to microscopic articular cartilage degeneration in guinea pigs with spontaneous osteoarthritis. Osteoarthritis Cartilage 21: 574-581, 2013.

30. Muraoka T, Hagino H, Okano T, Enokida M and Teshima R: Role of subchondral bone in osteoarthritis development: A comparative study of two strains of guinea pigs with and without spontaneously occurring osteoarthritis. Arthritis Rheum 56: 3366-3374, 2007

31. Uasnichka HL, Anderson-MacKenzie JM and Bailey AJ: Subchondral bone and ligament changes precede cartilage degradation in guinea pig osteoarthritis. Biorheology 43: 389-397, 2006.

32. Radin EL and Rose RM: Role of subchondral bone in the initiation and progression of cartilage damage. Clin Orthop Relat Res: 34-40, 1986.

33. Ding M and Hvid I: Quantification of age-related changes in the structure model type and trabecular thickness of human tibial cancellous bone. Bone 26: 291-295, 2000.
34. Ding M, Odgaard A, Danielsen CC and Hvid I: Mutual associations among microstructural, physical and mechanical properties of human cancellous bone. J Bone Joint Surg Br 84: 900-907, 2002.

35. Zhao W, Wang T, Luo Q, Chen Y, Leung VY, Wen C, Shah MF, Pan H, Chiu KY, Cao X and Lu WW: Cartilage degeneration and excessive subchondral bone formation in spontaneous osteoarthritis involves altered TGF- $\beta$ signaling. J Orthop Res 34: 763-770, 2016

36. van der Kraan PM: Factors that influence outcome in experimental osteoarthritis. Osteoarthritis Cartilage 25: 369-375, 2017.

37. Liu W, He J, Lin R, Liang J and Luo Q: Differential proteomics of the synovial membrane between bilateral and unilateral knee osteoarthritis in surgery-induced rabbit models. Mol Med Rep 14: 2243-2249, 2016.

38. Fellows CR, Matta $\mathrm{C}$ and Mobasheri A: Applying proteomics to study crosstalk at the cartilage-subchondral bone interface in osteoarthritis: Current status and future directions. EBioMedicine 11: 2-4, 2016.

39. Zhang R, Fang H, Chen Y, Shen J, Lu H, Zeng C, Ren J, Zeng H, Li Z, Chen S, et al: Gene expression analyses of subchondral bone in early experimental osteoarthritis by microarray. PLoS One 7: e32356, 2012

40. Andersen TL,Sondergaard TE,SkorzynskaKE,Dagnaes-HansenF, Plesner TL, Hauge EM, Plesner T and Delaisse JM: A physical mechanism for coupling bone resorption and formation in adult human bone. Am J Pathol 174: 239-247, 2009.

41. Shaw AT and Gravallese EM: Mediators of inflammation and bone remodeling in rheumatic disease. Semin Cell Dev Biol 49: 2-10, 2016.

42. Schelbergen RF, de Munter W, van den Bosch MH, Lafeber FP, Sloetjes A, Vogl T, Roth J, van den Berg WB, van der Kraan PM, Blom AB and van Lent PL: Alarmins S100A8/S100A9 aggravate osteophyte formation in experimental osteoarthritis and predict osteophyte progression in early human symptomatic osteoarthritis. Ann Rheum Dis 75: 218-225, 2016.

43. Anderson SL, Jalas C, Fedick A, Reid KF, Carpenter TO, Chirnomas D, Treff NR, Ekstein J and Rubin BY: A founder mutation in the TCIRG1 gene causes osteopetrosis in the Ashkenazi Jewish population. Clin Genet 88: 74-79, 2015.

44. Gheorghe G, Galambos C, Jain S, Krishnamurti L and Jaffe R: A novel TCIRG1 gene mutation leads to severe osteopetrosis with altered content of monocytes/macrophages in several organs. Pediatr Dev Pathol 15: 156-159, 2012.

45. Frattini A, Orchard PJ, Sobacchi C, Giliani S, Abinun M, Mattsson JP, Keeling DJ, Andersson AK, Wallbrandt P, Zecca L, et al: Defects in TCIRG1 subunit of the vacuolar proton pump are responsible for a subset of human autosomal recessive osteopetrosis. Nat Genet 25: 343-346, 2000.

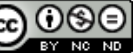

This work is licensed under a Creative Commons Attribution-NonCommercial-NoDerivatives 4.0 International (CC BY-NC-ND 4.0) License. 